P04-3-50 Poster session

\title{
Involvement of NADPH oxidase 1 in UVB-induced cell signaling and cytotoxicity in human keratinocytes
}

\author{
Azela Glady ${ }^{1}$, Manami Tanaka ${ }^{1}$, Catharina Sagita Moniaga ${ }^{1}$, Masato Yasui $^{1,2}$, Mariko Hara Chikuma ${ }^{1}$ \\ ${ }^{1}$ Pharmacology, Keio University, Japan, ${ }^{2}$ Keio Advanced Research Center for Water Biology and Medicine, Japan
}

Background: Members of NADPH oxidase (Nox) enzyme family are important sources of reactive oxygen species (ROS) and are known to be involved in several physiological functions in response to various stimuli including UV irradiation. UVB-induced ROS have been associated with inflammation, cytotoxicity, cell death, or DNA damage in human keratinocytes. However, the source and the role of UVB-induced ROS remain undefined.

Method: We measured intracellular ROS, cell viability, cytotoxicity and cell signaling in response to UVB radiation (15 $100 \mathrm{~mJ} / \mathrm{cm} 2)$ using human keratinocyte (HaCaT cell), which transfected with Nox1 or non-targeting-siRNA. We also determined ear swelling of C57BL/6 mice which administered intradermal injections of the cell lysate.

Result: Nox1 knockdown decreased UVB-induced ROS production in human keratinocytes. Moreover, Nox1 knockdown impaired UVB-induced p38 activation, accompanied by reduced IL-6 levels and attenuated cell toxicity. Treatment of cells with N-acetyl-L-cysteine (NAC), a potent ROS scavenger, suppressed p38 activation as well as consequent IL-6 production and cytotoxicity in response to UVB exposure. p38 inhibitor also suppressed UVB-induced IL-6 production and cytotoxicity. Furthermore, the blockade of IL- 6 production by IL- 6 neutralizing antibody reduced UVB-induced cell toxicity. Coincidentally, the intradermal injection of lysates from UVB-irradiated control cells, but not from UVBirradiated Nox1 knockdown cells, induced inflammatory swelling and IL-6 production in the skin of ears in wild-type mice.

Conclusion: Nox1-mediated ROS production is required for UVB-induced cytotoxicity and inflammation through p38 activation and inflammatory cytokine production, such as IL-6. Our findings suggest Nox1 as a therapeutic target for cytotoxicity and inflammation in response to UVB exposure. 